\title{
Covid-19: repercussões na saúde e no processo de trabalho dos agentes comunitários de saúde
}

\section{Covid-19: repercussions on the health and work process of community health agents}

Covid-19: repercusiones en la salud y lo proceso de trabajo de los agentes comunitarios de salud

Anne Louise de Souza Soares ${ }^{1 *}$, Adna Vivianne Rocha Freire ${ }^{1}$, Carlos Roberto Monteiro de Vasconcelos Filho ${ }^{1}$, Jessyca Alves das Neves Costa ${ }^{1}$, Alessa de França Cunha Medeiros ${ }^{1}$, Maria Elenilda do Milagre Alves dos Santos ${ }^{1}$, Jorgeane Pedrosa Pantoja ${ }^{1}$, Tatiane Bahia do Vale Silva ${ }^{1}$.

\section{RESUMO}

Objetivo: Investigar os impactos na saúde e a reorganização do processo de trabalho dos Agentes Comunitários de Saúde (ACS) de uma Estratégia Saúde da Família no Município de Belém que atuaram no enfrentamento da pandemia da COVID-19. Métodos: Estudo de caso com abordagem quanti-qualitativa do tipo exploratória, com 10 ACS. Abordou-se o perfil sociodemográfico e o processo de trabalho, relação com o território, amigos e/ou familiares e aspecto emocional durante a pandemia de covid-19. A coleta de dados foi realizada por meio de entrevista semiestruturada. Os dados obtidos foram analisados com auxílio do software Excel e IramuteQ. Resultados: A amostra maioria feminina (80\%), com idade média de 39 anos, ensino superior completo (40\%). Destes $80 \%$ foram afastados por algum motivo durante a pandemia, todos realizaram teste para covid, dos quais $60 \%$ positivaram para Covid-19. Observou-se mudanças no processo de trabalho, uso considerável de tecnologias de comunicação social no cuidado, quanto a aspectos emocionais que apresentaram abalos devido medo, ansiedade e insônia. Conclusão: Conclui-se que a pandemia apesar de pontos positivos como a fortificação do uso de mídias sociais, causou danos emocionais aos agentes de saúde, repercutindo negativamente no bem-estar emocional.

Palavras-chave: Atenção primária à saúde, Agentes comunitários de saúde, Infecções por coronavírus.

\begin{abstract}
Objective: To investigate the impacts on health and the reorganization of the working process of the Community Health Agents (CHA) of a Family Health Strategy in the Municipality of Belém that are not facing the COVID-19 pandemic. Methods: Case study with a quantitative-qualitative exploratory approach, with 10 $\mathrm{CHA}$. The sociodemographic profile and the work process, relationship with the territory, friends and/or family members and emotional aspect during the covid-19 pandemic were addressed. Data collection was performed through semi-structured interviews. The data obtained were analyzed using Excel and IramuteQ software. Results: The sample was mostly female ( $80 \%)$, with an average age of 39 years, complete higher education $(40 \%)$. Of these $80 \%$ were removed for some reason during the pandemic, all underwent testing for covid, of which $60 \%$ tested positive for Covid-19. Changes were observed in the working process, considerable use of social communication technologies in care, regarding emotional aspects that presented shocks due to fear, anxiety and insomnia. Conclusion: It is concluded that the pandemic, despite positive points such as the strengthening of the use of social media, caused emotional damage to health agents, negatively impacting emotional well-being.
\end{abstract}

Keywords: Primary health care, Community health workers, Coronavirus infections.

\section{RESUMEN}

Objetivo: Investigar los impactos en la salud biopsicosocial y la reorganización de los procesos de trabajo de los Agentes Comunitarios de Salud de una Estrategia de Salud de la Familia en la ciudad de Belém que actuaron en la lucha contra la pandemia COVID-19. Métodos: Estudio de caso con abordaje exploratorio

1 Universidade do Estado do Pará (UEPA), Belém - PA. *E-mail: soareslouise@hotmail.com 
cuantitativo-cualitativo, con 10 ACS. Se abordó el perfil sociodemográfico y el proceso de trabajo, relación con el territorio, amigos y/o familiares y aspecto emocional durante la pandemia de covid-19. La recolección de datos se realizó mediante entrevistas semiestructuradas. Los datos obtenidos se analizaron mediante el software Excel e IramuteQ. Resultados: La muestra fue mayoritariamente femenina (80\%), con una edad promedio de 39 años, estudios superiores completos (40\%). De estos, el $80 \%$ se eliminaron por alguna razón durante la pandemia, todos se sometieron a pruebas de covid, de los cuales el $60 \%$ dieron positivo para Covid-19. Se observaron cambios en el proceso de trabajo, considerable uso de las tecnologías de la comunicación social en el cuidado, en aspectos emocionales que presentaban choques por miedo, ansiedad e insomnio. Conclusión: Se concluye que la pandemia, a pesar de puntos positivos como el fortalecimiento del uso de las redes sociales, provocó daño emocional a los agentes de salud, impactando negativamente el bienestar emocional.

Palabras clave: Atención primaria de salud, Agentes comunitarios de salud, Infecciones por coronavirus.

\section{INTRODUÇÃO}

A pandemia da doença do coronavírus (COVID-19) afetou a saúde e o cotidiano de todos os sujeitos. Os profissionais da saúde sofreram, diretamente, com seus impactos. Diante de um período de crise sanitária causada pela pandemia, os níveis de atenção à saúde, estabelecidos pelo Sistema Único de Saúde (SUS) também apresentaram dificuldades para lidar com as problemáticas, entre elas, a Atenção Primária à Saúde (APS). Os profissionais da APS, além de sofrerem com a exposição direta ao vírus durante o contato com pacientes em atendimentos e visitas, também estão expostos a ausência de condições básicas de trabalho e o impacto do contexto pandêmico na saúde mental e social (SOEIRO RE, et al., 2020).

Entre os profissionais atuantes na APS e na equipe multidisciplinar, está o Agente Comunitário de Saúde (ACS). Os ACS's são profissionais que visam ofertar a promoção em saúde dentro de sua área adscrita. De acordo com a Portaria no 1886, de 18 de dezembro de 1997, este é o profissional que estabelece o vínculo entre a comunidade e o serviço de saúde, promovendo atividades de prevenção de doenças e promoção da saúde por meio de visitas domiciliares e atividades educativas individuais e em grupo nas famílias e comunidades (BRASIL, 1997).

O ACS é responsável por uma multiplicidade de atuações dentro do território, mantendo contato direto com os sujeitos que ali vivem. Esta exposição torna o profissional diretamente vulnerável a contrair, ser vetor, ou transmitir doenças, como no caso do COVID-19. Além disso, o ACS também está exposto as afecções mentais, emocionais e sociais causadas pela pandemia, como a necessidade de se manter no local de trabalho, por ser de um serviço essencial, o distanciamento social e também as possíveis perdas causadas pela morte e/ou quebra de vínculos impostas pelo contexto (SOEIRO RA, et al., 2020).

A pandemia piorou a saúde do agente, agravou sintomas como ansiedade, medo e insônia. Além disso, durante o período de pandemia alguns dos fatores intensificaram doenças físicas e emocionais nesses trabalhadores como a preocupação com o adoecimento e a morte, sensação de culpa por espalhar o vírus e contaminar familiares e pessoas próximas, a perda de colegas e outras pessoas devido à Covid-19, condições de trabalho instáveis, falta de EPI suficiente e falta de treinamento e informação do ACS para lidar com o vírus (NOGUEIRA ML, et al., 2021).

No município de Belém, de acordo com estudos publicados a cidade passou por duas ondas de pico do vírus, no ano de 2020 e 2021 durante os meses de abril e maio, com períodos de restrições rígidas para população, como "lockdown". Desde o início da pandemia diversos foram os relatos de profissionais da saúde da rede privada e pública em relação ao a condições de saúde e de trabalho durante a pandemia. De forma que o ACS em determinados momentos ainda transitava nas ruas para realização do seu trabalho mesmo sem as devidas condições (DEPARTAMENTO DE VIGILÂNCIA EM SAÚDE DE BELÉM, 2021).

Desta forma, o estudo objetivou investigar os impactos na saúde e a reorganização do processo de trabalho dos Agentes Comunitários de Saúde de uma Estratégia Saúde da Família no Município de Belém que atuaram no enfrentamento da pandemia da COVID-19. 


\section{MÉTODOS}

Estudo desenvolvido em uma dupla abordagem quanti-qualitativa do tipo exploratória. A coleta das informações foi realizada entre novembro e dezembro de 2021, a partir de entrevistas individuais, utilizando um roteiro semiestruturado elaborado pelas pesquisadoras dividido em duas partes: uma referente à identificação e informações quanto ao tempo de serviço, afastamento por COVID, realização de testagem COVID-19 e outra com cinco questões relacionadas a reorganização dos processos de trabalho diante da realidade da pandemia, percepções do ACS quanto à sua saúde física, mental e social e possíveis repercussões associadas ao processo da pandemia.

De acordo com Instituto Brasileiro de Geografia e Estatística (IBGE) o município de Belém possui estimativa populacional de 1.492 .745 habitantes em 2020, com uma cobertura de ESF aproximadamente de 23,11\%. Em 12/2020 o município contava com 600 ACS. A estimativa da população coberta pelos ACS era de 345.000 pessoas (SISTEMA DE INFORMAÇÃO EM SAÚDE PARA ATENÇÃO BÁSICA (SISAB), 2021). O local de desenvolvimento da pesquisa foi a Estratégia Saúde da Família (ESF), localizada no bairro de Valde-Cans, na qual pertence a Unidade Municipal de Saúde. A Estratégia é composta por 3 equipes, constituída por 27 ACS. A escolha da ESF se deu por conveniência devido os pesquisadores realizarem atividades na unidade.

A amostra foi composta por 10 ACS atuantes, as entrevistas foram encerradas ao atingir a saturação das informações, ou seja, quando as informações obtidas passaram a se repetir entre os entrevistados, e novas perspectivas e percepções sobre o mesmo tema deixaram de emergir, de forma que as entrevistas foram encerradas no $10^{\circ}$ participante.

Participaram do estudo os agentes comunitários de saúde atuantes na ESF que permaneceram atuantes durante os períodos de pico da pandemia da COVID-19 no Brasil e os que estivessem dispostos a participar da pesquisa. Foram excluídos do estudo os profissionais afastados do serviço durante o pico de novos casos da doença, por motivos de férias ou outras circunstâncias não relacionadas à pandemia, como afastamento devido ser do grupo de risco (comorbidades, idade).

As entrevistas foram gravadas em áudio, transcritas na íntegra e em seguida organizadas em um corpus textual, conjunto de texto que foi analisado, cada texto corresponde a um entrevistado. As perguntas da entrevista foram eliminadas, mas referenciadas, mantendo apenas as respostas completas. Cada entrevista foi dividida por uma linha de comando, contendo apenas uma variável $(\mathrm{N})$, seguido do número da ordem de entrevista, como por exemplo, segundo entrevistado (N2). Após realizada a transcrição das entrevistas, o texto foi salvo com caracteres no padrão "Unicode Transformation Format 8 bit codeunits".

Em seguida, todo o documento foi revisado, corrigido erros de digitação e pontuação e realizada a união palavras compostas, por exemplo, o termo "agente_comunitário_de_saúde", se incluído sem underline o sistema irá tratá-lo como se fossem quatro palavras diferentes. Por meio da análise do corpus, são geradas palavras com significância estatística, permitindo análise qualitativa dos dados.

Após a realização da coleta de dados, as informações para traçar o perfil dos ACS's foram sistematizadas por meio da digitalização e tabulação em banco de dados para, posteriormente, serem analisadas estatisticamente. Para os aspectos sociodemográficos foi realizada uma análise de dados descritiva, utilizouse o software Excel® 2010 para entrada dos dados, confecção das tabelas. O segundo momento da análise pesquisa foi qualitativa. Para isto, utilizou-se o software IraMuTeQ com a análise de Classificação Hierárquica Descendente (CHD).

A pesquisa foi submetida à apreciação do Comitê de Ética em Pesquisa da Universidade do Estado do Pará e aprovado (CAAE: 48638721.2.0000.5174, parecer 5.089.932) em conformidade com a Resolução do Conselho Nacional de Saúde 466/2012 e a 510/2016, e somente foi realizado após a assinatura de cada participante do Termo de Consentimento Livre e Esclarecido (TCLE). Com relação à garantia do anonimato, foi utilizado numerações para identificar os participantes, com vistas à proteção dos dados coletados. 


\section{RESULTADOS}

Neste estudo, $80 \%$ da amostra é do sexo feminino com idade média de 39 anos. Quanto ao nível de escolaridade $40 \%$ possui ensino superior completo, $90 \%$ dos entrevistados estão no cargo de ACS há mais de 6 anos. Foi possível perceber que a maioria dos participantes eram mulheres, o que reitera o perfil de feminização da área da saúde, principalmente entre os agentes comunitários de saúde. Os resultados da pesquisa com o grupo focal realizado pela Fiocruz corroboram com dados da amostra deste estudo. A pesquisa tinha o intuito de monitorar a saúde dos ACS em tempos de pandemia, no qual no primeiro grupo focal $100 \%$ dos participantes eram do sexo feminino com média de idade de 37 anos e a média de tempo de experiência profissional como ACS, foi de dez anos (NOGUEIRA ML, et al., 2021).

No que se refere à escolaridade e formação profissional, todos os participantes da pesquisa possuem ensino médio completo, dos 10 entrevistados, quatro têm curso superior completo e três estão cursando a graduação, demonstrando que os ACS possuem um nível de escolaridade contemplador ou superior ao exigido pelo Ministério da Saúde, Lei no 11.350/2006, que prevê a conclusão do ensino fundamental. O trabalho do agente comunitário, inicialmente, voltado para a educação em saúde, não possui a obrigação de conhecimento predominantemente técnico e científico, com o decorrer dos anos, houve mudança no perfil desses profissionais com crescente escolarização (SIMAS PRP e PINTO ICDM, 2017; AGUIAR RG, et al., 2018; GUIMARÃES MAS, et al., 2018).

Durante o período de pandemia, pelo menos $80 \%$ dos entrevistados foram afastados do serviço, destes $30 \%$ por infecção pelo coronavírus e $20 \%$ por suspeita de infecção pelo vírus. Todos os entrevistados realizaram teste para COVID-19, $80 \%$ realizaram teste rápido e $20 \%$ realizaram Rt-pcr, destes $40 \%$ testaram positivo (Tabela 1).

Tabela 1 - Afastamento dos Agentes comunitários de saúde participantes da pesquisa.

\begin{tabular}{|c|c|c|}
\hline Variável & $\mathbf{N}$ & $\%$ \\
\hline \multicolumn{3}{|l|}{ Tempo de afastamento (dias) } \\
\hline Menor ou igual a 30 dias & 7 & 70 \\
\hline $31-90$ dias & 3 & 30 \\
\hline$>90$ dias & 0 & 0 \\
\hline \multicolumn{3}{|l|}{ Causa do afastamento } \\
\hline Sem afastamento & 2 & 20 \\
\hline Infecção covid & 3 & 30 \\
\hline Suspeita covid & 2 & 20 \\
\hline Grupo de risco & 2 & 20 \\
\hline Outros & 1 & 10 \\
\hline \multicolumn{3}{|l|}{ Realizou teste para COVID-19 } \\
\hline Sim & 10 & 100 \\
\hline Não & 0 & 0 \\
\hline \multicolumn{3}{|c|}{ Tipo de testagem para COVID-19 } \\
\hline Teste rápido & 8 & 80 \\
\hline Rt-pcr & 2 & 20 \\
\hline \multicolumn{3}{|c|}{ Resultado teste para COVID-19 } \\
\hline Positivo & 4 & 40 \\
\hline Negativo & 6 & 60 \\
\hline Total & 100 & 100 \\
\hline
\end{tabular}

Fonte: Soares ALS, et al., 2021.

No estudo realizado com 884 ACS's no território brasileiro no período de junho a julho de 2020 observouse que $59,4 \%$ dos entrevistados apresentaram sinal ou sintoma de covid-19, 69,6\% tiveram acesso ao teste para covid-19 e dos que realizaram o teste tiveram resultado positivo, abrangendo $72,5 \%$ dos entrevistados (NOGUEIRA ML, et al., 2021). Estes achados corroboram com os encontrados neste estudo.

As classes lexicais receberam as seguintes nomeações: a) classe 1: Relação do ACS com a comunidade durante o período de pandemia; b) classe 2: relação do ACS com familiares e amigos durante a pandemia; $c$ ) classe 3: forma como a pandemia interferiu no processo de trabalho dos ACS's; d) classe 4: a pandemia interferiu no bem-estar emocional do ACS. 
No dendograma deste estudo, o corpus foi dividido em dois subcorpus. No primeiro, obteve-se a classe 4 que corresponde a $30,7 \%$ dos seguimentos de texto (ST). No segundo, obteve-se a classe 2,1 e 3 que houve uma segunda subdivisão, que englobou a classe 1 e a classe 2 , sendo $22,6 \%$ referente à classe 1 e $19,8 \%$ à classe 2 do total dos ST e a classe 3 correspondeu a 26,9\% do total (Figura 1).

Figura 1 - Dendograma com a porcentagem de UCE em cada classe fornecido pelo IraMUTeQ.

$$
\text { Corpus - INVESTIGAÇÃO DOS IMPACTOS NA SAÚDE BIOPSICOSSOCIAL E A REORGANIZAÇÃO }
$$
DOS PROCESSOS DE TRABALHO DOS AGENTES COMUNITÁRIOS DE SAÚDE

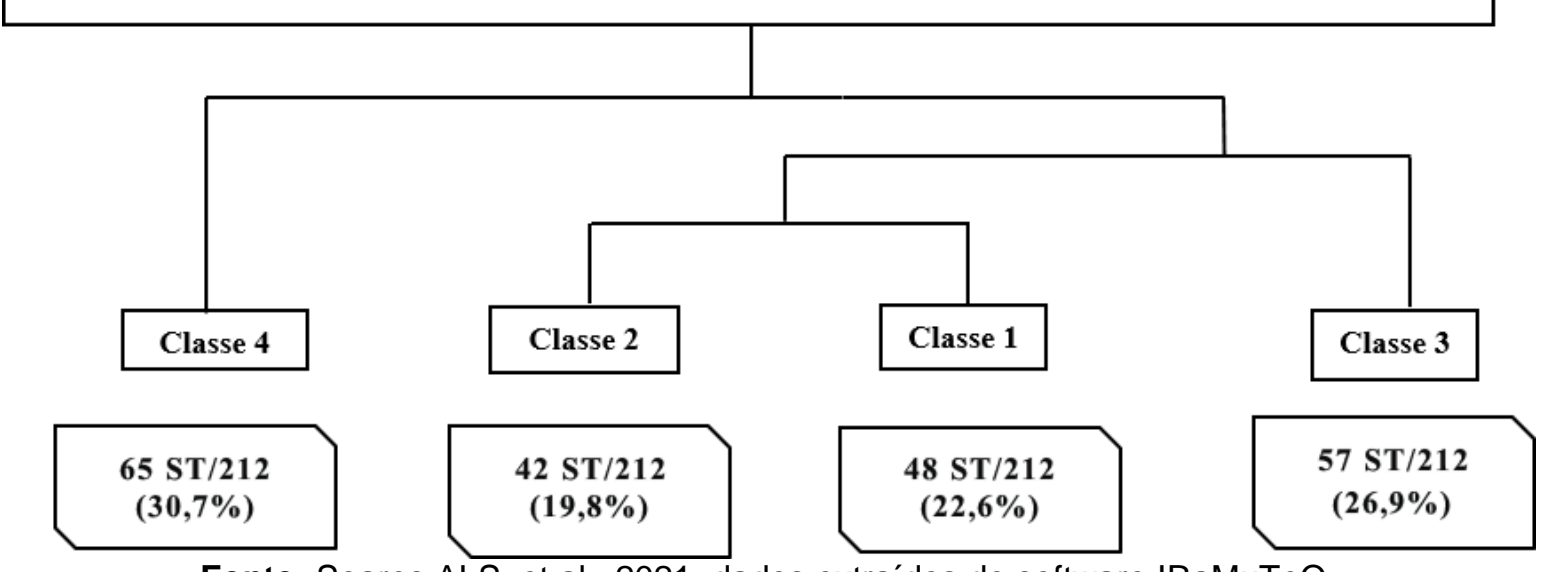

Fonte: Soares ALS, et al., 2021; dados extraídos do software IRaMuTeQ.

Após análise do software IraMuTeQ foram geradas palavras, posteriormente inseridas nos ST em que por meio destas o objetivo do estudo foi alcançado, voltado a verificar os impactos na saúde biopsicossocial e a reorganização dos processos de trabalho dos Agentes Comunitários de Saúde da Estratégia Saúde da Família do Município de Belém que atuam no enfrentamento da pandemia da COVID-19.

A classe 4 demonstra esses aspectos emocionais que acometem os ACS durante o período pandêmico. Nomeada como "Interferência da pandemia no bem-estar emocional do ACS", a mesma incorporou 30,7\% dos segmentos de textos, as principais palavras que formaram a classe foram: físico, melhorar, bastante, começar, situação, comunidade, psicóloga e pandemia. O conteúdo está relacionado às mudanças psicológicas, abalos emocionais adquiridos durante o pico de covid-19. Os trechos a seguir ilustram esse contexto:

"Não só a mim, mas eu acredito que a grande parte dos agentes de saúde, ficaram bem abalados com essa situação da pandemia tanto que grande parte acabou utilizando medicação essa questão do pânico" (N1).

"Apesar da pandemia o afastamento do trabalho me ajudou bastante melhorou 0 estresse sem cobranças as crises de ansiedade diminuíram" (N3).

"E eu acho assim que ansiedade foi uma das causas assim que eu observei nas pessoas vi muitas pessoas procurarem até mandar um WhatsApp pra mim me contando essa internet ajudou também essa questão" (N8).

Resultados semelhantes foram encontrados ao pesquisar a literatura sobre estudos feitos sobre o impacto na saúde mental das pessoas durante a pandemia. Acredita-se que os sintomas psiquiátricos tenham se tornado mais fortes na sociedade em geral, mas especialmente entre os profissionais de saúde que foram direta ou indiretamente expostos ao contágio (ORNEL F, et al., 2020; OLIVEIRA BV, et al., 2021).

O estudo realizado pela Fiocruz (2020) aborda o sofrimento emocional do agente de saúde. Dos entrevistados, $23 \%$ perderam um familiar, amigo e/ou colega de trabalho durante a pandemia, mais de $80 \%$ se sentiram emocionalmente abalados. E em relação aos aspectos emocionais relacionados ao abalo emocional, apresentaram sintomas como ansiedade (63\%), angústia (60,6\%), insônia (48,8\%), medo (45,1\%), entre outros. Tais achados corroboram com as falas dos entrevistados deste estudo. 
A classe 1 nomeada "Relação do ACS com a comunidade durante o período de pandemia" foi responsável por $22,6 \%$ dos segmentos de texto. O conteúdo destacado trata principalmente da maneira que os usuários do território que o ACS era responsável estavam se relacionando/comunicando com o mesmo durante 0 período de pandemia, principalmente durante o período de "Lockdown".

Tendo em vista a necessidade de se adotar medidas de distanciamento social e restringir a realização de visita domiciliar, em circunstâncias especiais, a possibilidade e a ética do uso da telemedicina são consideradas prioritárias para a continuação do cuidado do ACS com o usuário. Os trechos a seguir ilustram esse contexto:

"E agora mais por WhatsApp eu vou na casa, mas pra levar uma consulta ou então peço pro usuário ou responsável vir na unidade pra mim não ter muito contato com eles" (N3).

"Porque eu já que quando eles precisavam eles mandavam um WhatsApp eu espalhei meu WhatsApp para os usuários isso ajudou muito, porque ajudavam a confortar eu sempre mandava informações como era pra evitar" (N8).

O principal meio de promoção de saúde do ACS é por meio da visita domiciliar, porém com o advento da pandemia a mesma sofreu modificações. De acordo com recomendações do Ministério da Saúde (2020) aos ACS as visitas domiciliares não deveriam ocorrer dentro dos domicílios, mas nas áreas peridomiciliares (frente, lados e fundo do quintal ou terreno). De acordo com um estudo realizado entre 28 de maio e 3 de julho de $2020 \mathrm{com} 1.978$ ACSs, $17 \%$ das visitas domiciliares foram suspensas, 54,7\% continuaram em regime reduzido e apenas $20,9 \%$ não relataram alteração durante a visita (SECRETARIA DA SAÚDE DE SÃO PAULO, 2020; MÉLLO LMBD, et al., 2021).

A partir do momento da suspensão/redução das visitas, estratégias de aproximação do ACS com os usuários tiveram que ser traçadas, dentre elas o uso de Tecnologias de Informação e Comunicação (TICs). Estratégias de comunicação e mobilização comunitária são importantes para adequação do trabalho do ACS na situação da pandemia como: a realização do contato com usuário por meio dos canais de comunicação tais como: whatsapp, email, telefone, entre outros outros, para monitorar, informar o público sobre o cancelamento de agendamentos e nortear a cerca da agenda de trabalho da unidade (JUNIOR NB, et al., 2020; BARBOSA MS, et al., 2021).

O uso de aplicativos móveis como redes sociais e WhatsApp, entende-se que tornou-se uma realidade na prática profissional, e mesmo que não seja institucionalizado, é necessário ampliar o acesso à tecnologia e conexões para fazer os trabalhadores elegíveis para o melhor aproveitamento de ferramentas no âmbito da comunicação social e vigilância da saúde. $O$ fato de uma em cada quatro pessoas no Brasil não conseguir acessar a Internet revela a nova equidade do SUS e a importância da realização de visitas domiciliares de ACS(NÚCLEO DE INFORMAÇÃO E COORDENAÇÃO DO PONTO BR, 2020; MACIEL FBM, et al., 2020).

A classe 2 "Relação do ACS com familiares e amigos durante a pandemia" incorporou $19,8 \%$ dos segmentos de textos, as principais palavras que formaram a classe foram: sentir, nada, sair, mundo, positivo, não, chegar, próximo, abraçar. O conteúdo está relacionado à forma com que os ACS se sentiram em relação à interação social com familiares e amigos durante o pico do coronavírus. Os trechos a seguir ilustram esse contexto:

"Onde dentro de casa a gente brincava fazia ligações se divertia comia a gente teve que se fechar naquele mundo então mudou muito" (N2).

"Olha eu vivo normal agora no momento claro a gente não tem mais aquela pegação de ficar grudado, não existe isso a gente sempre tem que ter o cuidado respeito" (N4).

"O paraense ele já tem aqueles acolhimentos de querer agarrar, querer abraçar, querer beijar, eu sou assim, aí eu chegava aqui eu não via ninguém, às vezes eu chegava olhava alguém e não podia abraçar não podia beijar porque eu sabia que eu tinha que chegar em casa, não pegar nada pra não levar pra casa, aí foi difícil" (N7). 
A classe 3 "Forma como a pandemia interferiu no processo de trabalho dos ACS's" incorporou 26,9\% dos segmentos de textos, as principais palavras que formaram a classe foram: paciente, contato, criança, receber, distanciamento, criar, irmão e emocional. O conteúdo está relacionado à diferença de tratamento da comunidade, percebida pelos ACS's, em relação ao processo de trabalho durante os períodos de pico de novas infecções do coronavírus. Os trechos a seguir ilustram esse contexto:

"Eu senti na área foi o medo das pessoas de nos receber de me receber na residência principalmente as pessoas que são no grupo de risco elas não permitiram que eu adentrasse a residência delas" (N2).

"A gente tá muito em contato com os pacientes com os usuários que a gente chama na nossa área, então isso realmente pesou um certo impacto, porque devido a aproximação que a gente tem, nessa relação, então isso dificultou porque a gente tinha que manter distância e tem pessoas que realmente o ser humano é um contato ele quer abraçar cumprimentar" (N8).

"Mas a questão que a gente não podia entrar na casa dos usuários tinha que ser na porta e tinha uns que nem recebiam e tinha uns que eu também não queria nem entrar por conta do medo de eu transmitir se tivesse passando" (N9).

As classes 2 e 3 como mostra o dendograma possuem uma ligação, isto pode ser explicado devido o aspecto emocional possuir uma transversalidade nas classes. O medo de contaminação tanto pelos familiares e amigos como pelos usuários do SUS que eles fazem cobertura. Na linha de frente da política de saúde, as ACS são atrizes chave para atuar no enfrentamento de crises sanitárias, como é o caso da pandemia de COVID-19, dado o seu amplo conhecimento sobre as limitações e as potências do território em que atuam e a proximidade com as famílias acompanhadas (LOTTA G, et al., 2020). Os agentes comunitários desempenham um papel importante no SUS, aproximando cada vez mais as pessoas do sistema público de saúde.

Durante a pandemia o ACS passou a ser visto pela comunidade como alguém que ao mesmo tempo que protegia, causava medo. O medo no território era observado nas recusas da visita domiciliar do agente. Com base nos dados da pesquisa realizada pela Fiocruz (2020) no território nacional, os resultados demonstraram que na própria vizinhança o profissional de saúde sofreu algum tipo de discriminação, aproximadamente 34\% ou durante o caminho percorrido trabalho/casa, representando $28 \%$. Pode-se inferir, que a população viu o profissional de saúde como um disseminador do vírus, logo, considerava o mesmo um risco.

Além do medo da comunidade, o ACS carregava consigo o medo de contaminação de seus familiares, por conta de os mesmos estarem na parte dianteira de combate ao coronavírus, sempre na unidade fazendo serviço remoto ou dando assistência aos usuários que iam em busca de atendimento. Durante os relatos, foi possível observar que os familiares dos agentes de saúde tiveram que viver com vários sentimentos, incluindo preocupações e medos, principalmente pelo fato de ser uma doença pouco conhecida, sem muitos relatos. Além disso, eles estão preocupados que seus familiares, também profissionais de saúde, fossem infectados durante o trabalho ou levassem o vírus para casa e infectassem outros membros da família.

Durante a pandemia, rastreamos as preocupações de toda a comunidade sobre o impacto da saúde mental das pessoas, incluindo isolamento, distanciamento social, suspensões de escolas, o fechamento de locais comerciais que comprovaram a crise, o impacto do aumento de casos, entre muitos outros fatores (FARO A, et al., 2020). Esses agravos à saúde mental têm sido previstos pelos profissionais de saúde, que estão na linha de frente, recebendo, acolhendo, cuidando e acompanhando pessoas que suspeitam ou confirmam o COVID-19 e buscam constantemente o desenvolvimento da resiliência (DANTAS ESO, 2021).

Estes agravos foram percebidos por altos níveis de ansiedade, dor, estresse, medo, depressão, distúrbios do sono, etc. Esses sintomas mentais podem causar outros danos físicos aos trabalhadores, embora esses problemas estejam presentes na saúde ocupacional há muitos anos, essa situação intensificou-se com a eclosão da pandemia (PRADO AD, et al., 2020). 


\section{CONCLUSÃO}

A partir dos resultados deste estudo foi possível compreender os impactos na saúde e a reorganização do processo de trabalho dos ACS de uma ESF no Município de Belém que atuaram no enfrentamento da pandemia da COVID-19. Entende-se que as repercussões foram marcadas de maneira muito forte pela apreensão e medo decorrentes do surgimento de uma doença pouco conhecida e com rápida disseminação e de se ter um familiar na linha de frente; pelas mudanças nas relações familiares e no convívio domiciliar. Dessa maneira, devido à sobrecarga depositada no agente comunitário de saúde durante a pandemia, devese acompanhar este profissional, dando apoio psicológico e gerenciar políticas que possibilitem gerenciar os dados adquiridos e o processo de trabalho ajustado da melhor maneira possível.

\section{REFERÊNCIAS}

1. AGUIAR RG, et al. Qualidade de vida no trabalho do agente comunitário de saúde. Caderno de Educação, Saúde e Fisioterapia, 2018; 5(9): 42-51.

2. BARBOSA MS, et al. Fatores sociodemográficos e ocupacionais associados aos sintomas de ansiedade entre Agentes Comunitários de Saúde. Ciência \& Saúde Coletiva. 2021; 26(12): 5997- 6004.

3. BRASIL. MINISTÉRIO DA SAÚDE. Coronavírus COVID-19. 2020. Disponível em: https://coronavirus.saude.gov.br/. Acessado em: 26 de outubro de 2020.

4. BRASIL. Portaria no. 1.886, de 18 de dezembro de 1997. Aprova as normas e diretrizes do Programa de Agente Comunitário e do Programa de Saúde da Família e dá outras providências. Diário Oficial da União, Brasília, DF, 22 dez. 1997. Disponível em: https://bvsms.saude.gov.br/bvs/saudelegis/gm/2017/prt2436_22_09_2017.html. Acessado em: 15 de dezembro de 2021.

5. DANTAS, ESO. Saúde mental dos profissionais de saúde no Brasil no contexto da pandemia por Covid-19. Interface (Botucatu). 2021; 25 (1): e200203.

6. DEPARTAMENTO DE VIGILÂNCIA EM SAÚDE DE BELÉM. Boletim Epidemiológico - BELÉM. 2021. Disponível em: http://ww4.belem.pa.gov.br/wp-content/uploads/2021/01/31-ANEXO-BOLETIMEPIDEMIOL\%C3\%93GICO_COVID19_BEL\%C3\%89M_03_01_2021_Base_31_12_2020.pdf. Acessado em: 29 dez. 2021.

7. FARO A, et al. COVID-19 e saúde mental: a emergência do cuidado. Estudos de Psicologia. 2020; 37: e200074.

8. GUIMARÃES MAS, et al. Perfil sociodemográfico dos agentes comunitários de Saúde da Estratégia Saúde da Família no município de Palmas - TO. DESAFIOS - Revista Interdisciplinar da Universidade Federal do Tocantins. 2017; 4(3): 60-72.

9. JUNIOR NB, et al. Guia Orientador para o enfrentamento da pandemia Covid-19 na Rede de Atenção à Saúde. 2020. Disponível em: https://www.conasems.org.br/wp-content/uploads/2020/05/Instrumento-OrientadorConass-Conasems-VERS\%C3\%83O-FINAL-3.pdf. Acessado em: 13 de dezembro de 2021.

10. LOTTA G, et al. How COVID-19 Has Affected Frontline Workers in Brazil: A Comparative Analysis of Nurses and Community Health Workers. Journal of Comparative Policy Analysis: Research and Practice. 2020; 23(1): 63-73.

11. MACIEL FBM, et al. Agente comunitário de saúde: reflexões sobre o processo de trabalho em saúde em tempos de pandemia de Covid-19. Ciência \& Saúde Coletiva. 2020; 25(suppl 2): 4185-4195.

12. MÉLLO LMBD, et al. Agentes comunitárias de saúde: práticas, legitimidade e formação profissional em tempos de pandemia de Covid-19 no Brasil. Interface (Botucatu). 2021; 25(Supl. 1): e210306.

13. NOGUEIRA ML, et al. 3ํㅡㄹ Boletim da Pesquisa Monitoramento da saúde e contribuições ao processo de trabalho e à formação profissional dos Agentes Comunitários de Saúde em tempos de Covid-19. Rio de Janeiro: EPSJV/Fiocruz. Janeiro 2021. 68p.

14. NÚCLEO DE INFORMAÇÃO E COORDENAÇÃO DO PONTO BR. Pesquisa sobre o uso das tecnologias de informação e comunicação nos domicílios brasileiros: TIC Domicílios 2019. Comitê Gestor da Internet no Brasil. 2020. Disponível em: https://cetic.br/pt/publicacao/resumo-executivo-pesquisa-sobre-o-uso-das-tecnologias-de-informacaoe-comunicacao-nos-domicilios-brasileiros-tic-domicilios-2020/. Acessado em: 15 de dezembro de 2021.

15. OLIVEIRA BV, et al. Impacto da pandemia do COVID-19 sob o cuidado na atenção primária í saúde: percepção de enfermeiros. Saúde Coletiva (Barueri). 2021; 11(COVID): 7057-7072.

16. ORNEL F, et al. Pandemia de medo e Covid-19: impacto na saúde mental e possíveis estratégias. Debates em Psiquiatria. 2020; 10(2): 12-6.

17. PRADO AD, et al. A saúde mental dos profissionais de saúde frente à pandemia do COVID-19: uma revisão integrativa. Revista Eletrônica Acervo Saúde. 2020; (46): e4128.

18. SECRETARIA DA SAÚDE DE SÃO PAULO. Orientações para a organização das ações no manejo do novo coronavírus (COVID-19) na atenção primária à saúde (versão 2). 2020. Disponível em: https://docs.bvsalud.org/biblioref/2020/04/1087808/versao_dia_9_de_abril_orientacoes_para_a_organizacao_da_ate nca 7Q1g16X.pdf. Acessado em: 26 de outubro de 2020.

19. SIMAS PRP, PINTO ICDM. Trabalho em saúde: retrato dos agentes comunitários de saúde da região Nordeste do Brasil. Ciência \& Saúde Coletiva. 2017; 22: 1865-1876.

20. SISTEMA DE INFORMAÇÃO EM SAÚDE PARA A ATENÇÃO BÁSICA (SISAB). Painel de Indicadores referentes a Agentes Comunitários de Saúde. Disponível em: https://sisaps.saude.gov.br/painelsaps/acs. Acessado em: 15 de dezembro de 2021.

21. SOEIRO RE, et al. Atenção Primária à Saúde e a pandemia de COVID-19: reflexão para a prática. InterAmerican Journal of Medicine and Health. 2020; 3(7): e202003010. 